\title{
Article
}

\section{On 3-total edge product cordial labeling of tadpole, book and flower graphs}

\author{
Umar Ali $^{1}$, Yasir Ahmad ${ }^{1}$ and Muhammad Shoaib Sardar ${ }^{1, *}$ \\ 1 School of Mathematical Sciences, Anhui University, Hefei, Anhui, 230601, P.R. China.; \\ umarali.msc@gmail.com(U.A); yasirahmad6667@gmail.com(Y.A)
}

Received: 29 July 2019; Accepted: 13 February 2020; Published: 7 March 2020.

Abstract: In this paper, we will determine the 3-total edge product cordial (3-TEPC) labeling. We study certain classes of graphs namely tadpole, book and flower graphs in the context of 3-TEPC labeling.

Keywords: 3-total edge product cordial labeling, book graphs, flower graphs.

MSC: $05 C 78$.

\section{Introduction}

$\mathbf{W}$ e define with simple, finite and undirected graph $G=\left(E_{G}, V_{G}\right)$, where $E_{G}$ and $V_{G}$ is vertex set and edge set respectively. Cordinalities of there sets are called the size and order of $G$. We follow the standard notations and terminology of graph theory as in [1]. A graph labeling is an assignment to vertices or edges or both subject to certain conditions. If the domain is $V_{G} \cup E_{G}$ then we called the labeling is total labeling.

We have the following notations, in order to know cordial labeling $h$ and its sorts.

1. $v_{h}(j)$ is the number of vertices labeled by $j$;

2. $e_{h}(j)$ is the number of edges labeled by $j$;

3. $v_{h}(i, j)=v_{h}(i)-v_{h}(j)$;

4. $e_{h}(i, j)=e_{h}(i)-e_{h}(j)$ and

5. the sum of all vertices and edges labeled by $j$ is $\operatorname{sum}(j)$ i.e. $\operatorname{sum}(j)=v_{h}(j)+e_{h}(j)$.

Cahit [2] gave first concept of cordial labeling as a weaker version of graceful labeling. A vertex labeling $h: V_{G} \rightarrow\{0,1\}$ that induce an edge labeling $h^{*}: E_{G} \rightarrow\{0,1\}$ defined by $h^{*}(u v)=|h(u)-h(v)|$, for all $u, v \in E_{G}$ if $\left|v_{h}(1)-v_{h}(0)\right| \leq 1$ and $\left|e_{h}(1)-e_{h}(0)\right| \leq 1$. The concept of product cordial labeling was introduced by Sundaram et al. in 2014. For details see [3]. In 2006 and 2012, Sundaram et al. develop the concept of total product cordial labeling and $k$-total product cordial labeling. For details see $[4,5]$. Vaidya and Barasara gave the concept of edge product cordial labeling and total edge product cordial labeling. For details see [6,7].

Let $k$ be an integer, $2 \leq k \leq\left|E_{G}\right|$ an edge labeling $h: E_{G} \longrightarrow\{0,1, \ldots, k-1\}$, with induced vertex labeling $h^{*}: V_{G} \longrightarrow\{0,1, \ldots, k-1\}$ such that $h^{*}(u)=h\left(e_{1}\right) h\left(e_{2}\right) \ldots h\left(e_{n}\right)(\bmod k)$, where edges $e_{1}, e_{2}, \ldots, e_{n}$ are the edge incident to $u$, then $h$ is called $k$-total edge product cordial labeling if $|\operatorname{sum}(i)-\operatorname{sum}(j)| \leq 1$ for $i, j \in\{0,1, \ldots, k-1\}$.

In 2015, Azaizeh et al. [8] was introduced the basic concept of $k$-total edge product cordial ( $k$-TEPC) labeling. Recently Azaizeh et al. investigated the 3-TEPC labeling for more families of graphs namely, path, circle, fan, double fan, triangular snake graph (see example [9,10]). Ahmad et al. [11] discussed 3-TEPC labeling of gear, web and helm graph. Ali et al. [12] investigated the 3-TEPC labeling for families of convex polytopes namely, double antiprism $A_{m}, S_{m}$ and $T_{m}$. In 2018, Ahmad et al. [13] had discussed the 3-TEPC labeling for hexagonal grid. Recently, Ali et al. [14] investigated the 4-total edge product cordial labeling of some standard graphs. For more details see references [15-18].

Now we will define tadpole graph $\left(T_{p, q}\right)$, book graph $\left(B_{q}\right)$ and flower graph $\left(F l_{q}\right)$ graph.

Definition 1. The tadpole graph is obtained by connecting a cycle graph $C_{p}$ (of order $p$ ) to a path graph $P_{q}$ (of order q) with a bridge and is denoted by $T_{p, q}$ (see Figure 1). 


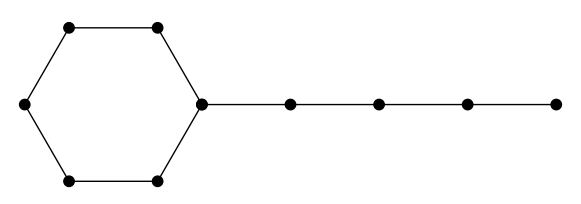

Figure 1. Tadpole graph $T_{6,4}$.

Definition 2. Book graph $B_{q}$ is obtained by the cartesian product $S_{q+1} \times P_{2}$, where $S_{q+1}$ is the star graph of order $q+1$ and $P_{2}$ is the path graph of order 2 (see Figure 2).

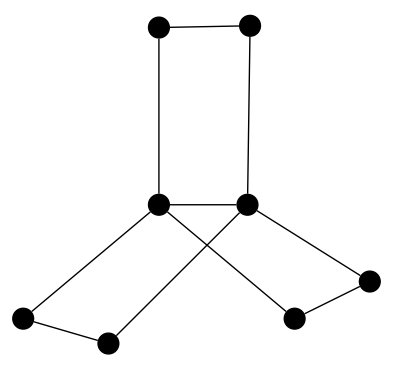

Figure 2. Book graph $B_{3}$

Definition 3. The flower graph $F l_{q}$ is the graph attain from a helm graph $H_{q}$ of order $q$ by joining each pendent vertex to the apex of the helm (see Figure 3).

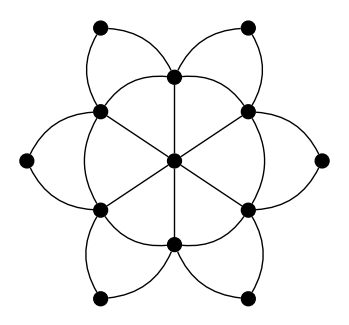

Figure 3. Flower graph $\mathrm{Fl}_{6}$

\section{Main results}

In this section, we will discuss 3-total edge product cordial (3-TEPC) labeling of tadpole, book and flower graphs.

Theorem 1. The tadpole graph $T_{p, q}$ has 3-TEPC labeling.

Proof. Let $V_{G}=\left\{u_{j}, 1 \leq j \leq p\right\} \cup\left\{v_{j}, 1 \leq j \leq q\right\}$ and $E_{G}=\left\{u_{j} u_{j+1}, 1 \leq j \leq p-1\right\} \cup\left\{v_{j} v_{j+1}, 1 \leq j \leq\right.$ $q-1\} \cup\left\{u_{p} u_{1}\right\} \cup\left\{v_{q} u_{p}\right\}$ as shown in Figure 1. Now we see the following three cases:

Case 1 Let $p+q \equiv 0(\bmod 3)$ which implies $p+q=3 t$, for $t \geq 3$. So for the given case, we need to discuss the following three subcases:

Case 1.1 If $p=0(\bmod 3)$ and $q=0(\bmod 3)$. We define $h: E_{G} \rightarrow\{0,1,2\}$ as:

$$
\begin{aligned}
& h\left(u_{j} u_{j+1}\right)=\left\{\begin{array}{ll}
0, & \text { if } 1 \leq j \leq \frac{p}{3}-1 ; \\
2, & \text { if } \frac{p}{3} \leq j \leq p-1 ;
\end{array} \text { and } h\left(u_{p} u_{1}\right)=1 .\right. \\
& h\left(v_{j} v_{j+1}\right)=\left\{\begin{array}{ll}
0, & \text { if } 1 \leq j \leq \frac{q}{3} ; \\
2, & \text { if } \frac{q}{3}+1 \leq j \leq q-1 .
\end{array}, h\left(v_{q} u_{p}\right)=2 .\right.
\end{aligned}
$$

Case 1.2 If $p>q$ but $p \neq 0(\bmod 3)$ and $q \neq 0(\bmod 3)$. We define $h: E_{G} \rightarrow\{0,1,2\}$ as: 


$$
\begin{aligned}
& h\left(u_{j} u_{j+1}\right)=\left\{\begin{array}{ll}
0, & \text { if } 1 \leq j \leq t-2 ; \\
2, & \text { if } t-1 \leq j \leq p-1 ;
\end{array} \text { and } h\left(u_{p} u_{1}\right)=1 .\right. \\
& h\left(v_{j} v_{j+1}\right)=\left\{\begin{array}{ll}
0, & \text { if } j=1 ; \\
2, & \text { if } 2 \leq j \leq q-1 .
\end{array}, h\left(v_{q} u_{p}\right)=2 .\right.
\end{aligned}
$$

Case 1.3 If $p<q$ but $p \neq 0(\bmod 3)$ and $q \neq 0(\bmod 3)$. We define $h: E_{G} \rightarrow\{0,1,2\}$ as:

$$
\begin{aligned}
& h\left(u_{j} u_{j+1}\right)=\left\{\begin{array}{ll}
0, & \text { if } j=1 ; \\
2, & \text { if } 2 \leq j \leq p-1 ;
\end{array} \text { and } h\left(u_{p} u_{1}\right)=1 .\right. \\
& h\left(v_{j} v_{j+1}\right)=\left\{\begin{array}{ll}
0, & \text { if } 1 \leq j \leq t-2 ; \\
2, & \text { if } t-1 \leq j \leq q-1 .
\end{array}, h\left(v_{q} u_{p}\right)=2 .\right.
\end{aligned}
$$

So we obtain $\operatorname{sum}(0)=\operatorname{sum}(1)=\operatorname{sum}(2)=2 t$. Thus $\left|\operatorname{sum}\left(x_{1}\right)-\operatorname{sum}\left(x_{2}\right)\right| \leq 1$ for $0 \leq x_{1}<x_{2} \leq 2$. Hence $h$ is 3-TEPC labeling as discussed in Figure 4.

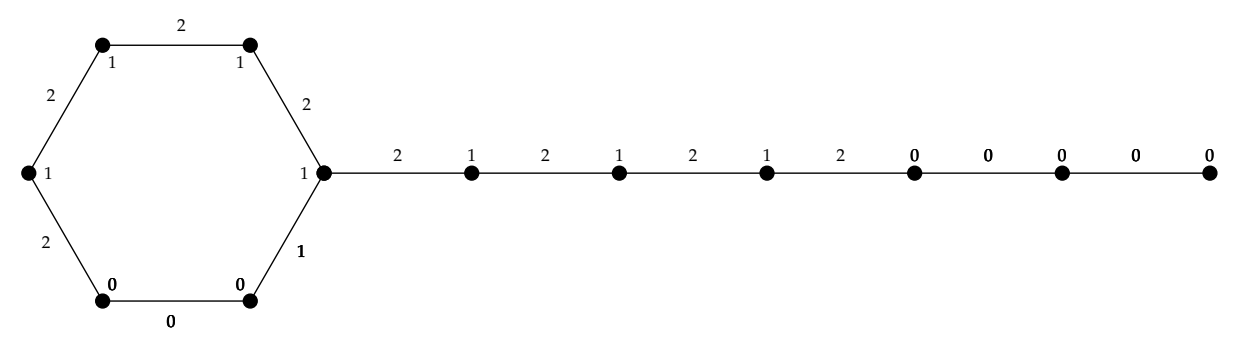

Figure 4. 3-TEPC labeling of $T_{6,6}$

Case 2 Let $p+q \equiv 1(\bmod 3)$ which implies $p+q=3 t+1$, for $t \geq 3$. So for the given case, we need to discuss the following three subcases:

Case 2.1 If $p=0(\bmod 3)$ and $q \neq 0(\bmod 3)$. We define $h: E_{G} \rightarrow\{0,1,2\}$ as:

$$
\begin{aligned}
& h\left(u_{j} u_{j+1}\right)=\left\{\begin{array}{ll}
0, & \text { if } 1 \leq j \leq \frac{p}{3}-1 ; \\
2, & \text { if } \frac{p}{3} \leq j \leq p-1 ;
\end{array} \text { and } h\left(u_{p} u_{1}\right)=1 .\right. \\
& h\left(v_{j} v_{j+1}\right)=\left\{\begin{array}{ll}
0, & \text { if } 1 \leq j \leq \frac{q-1}{3} ; \\
2, & \text { if } \frac{q-1}{3}+1 \leq j \leq q-1 .
\end{array}, h\left(v_{q} u_{p}\right)=2 .\right.
\end{aligned}
$$

Case 2.2 If $p \neq 0(\bmod 3)$ and $q=0(\bmod 3)$. We define $h: E_{G} \rightarrow\{0,1,2\}$ as:

$$
\begin{aligned}
& g\left(u_{j} u_{j+1}\right)=\left\{\begin{array}{ll}
0, & \text { if } 1 \leq j \leq \frac{p-1}{3}-1 ; \\
2, & \text { if } \frac{p-1}{3} \leq j \leq p-1 ;
\end{array} \text { and } h\left(u_{p} u_{1}\right)=1 .\right. \\
& h\left(v_{j} v_{j+1}\right)=\left\{\begin{array}{ll}
0, & \text { if } 1 \leq j \leq \frac{q}{3} ; \\
2, & \text { if } \frac{q}{3}+1 \leq j \leq q-1 .
\end{array}, h\left(v_{q} u_{p}\right)=2 .\right.
\end{aligned}
$$

Case 2.3 If $p \neq 0(\bmod 3)$ and $q \neq 0(\bmod 3)$. We define $h: E_{G} \rightarrow\{0,1,2\}$ as:

$$
\begin{aligned}
& h\left(u_{j} u_{j+1}\right)=\left\{\begin{array}{ll}
0, & \text { if } 1 \leq j \leq \frac{p-2}{3} ; \\
2, & \text { if } \frac{p-2}{3}+1 \leq j \leq p-1 ;
\end{array} \text { and } h\left(u_{p} u_{1}\right)=1 .\right. \\
& h\left(v_{j} v_{j+1}\right)=\left\{\begin{array}{ll}
0, & \text { if } 1 \leq j \leq \frac{q-2}{3} ; \\
2, & \text { if } \frac{q-2}{3}+1 \leq j \leq q-1 .
\end{array}, h\left(v_{q} u_{p}\right)=2 .\right.
\end{aligned}
$$

So we obtain $\operatorname{sum}(0)=2 t, \operatorname{sum}(1)=\operatorname{sum}(2)=2 t+1$. Thus $\left|\operatorname{sum}\left(x_{1}\right)-\operatorname{sum}\left(x_{2}\right)\right| \leq 1$ for $0 \leq x_{1}<x_{2} \leq$ 2. Hence $h$ is 3-TEPC labeling as discussed in Figure 5. 


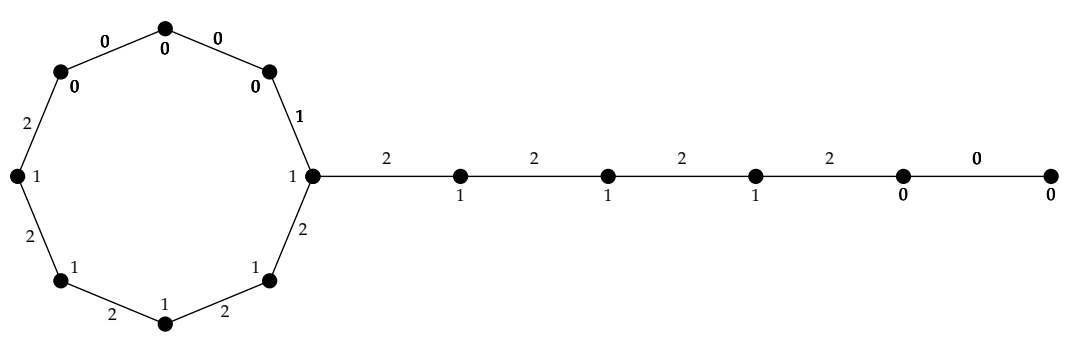

Figure 5. 3-TEPC labeling of $T_{8,5}$

Case 3 Let $p+q \equiv 2(\bmod 3)$ which implies $p+q=3 t+2$, for $t \geq 3$. So for the given case, we need to discuss the following three subcases:

Case 3.2 If $p=0(\bmod 3)$ and $q \neq 0(\bmod 3)$. We define $h: E_{G} \rightarrow\{0,1,2\}$ as:

$$
\begin{aligned}
& h\left(u_{j} u_{j+1}\right)=\left\{\begin{array}{ll}
0, & \text { if } 1 \leq j \leq \frac{p}{3} ; \\
2, & \text { if } \frac{p}{3}+1 \leq j \leq p-1 ;
\end{array} \text { and } h\left(u_{p} u_{1}\right)=1 .\right. \\
& h\left(v_{j} v_{j+1}\right)=\left\{\begin{array}{ll}
0, & \text { if } 1 \leq j \leq \frac{q-2}{3} ; \\
2, & \text { if } \frac{q-2}{3}+1 \leq j \leq q-1 .
\end{array}, h\left(v_{q} u_{p}\right)=2 .\right.
\end{aligned}
$$

Case 3.2 If $p \neq 0(\bmod 3)$ and $q=0(\bmod 3)$. We define $h: E_{G} \rightarrow\{0,1,2\}$ as:

$$
\begin{aligned}
& h\left(u_{j} u_{j+1}\right)=\left\{\begin{array}{ll}
0, & \text { if } 1 \leq j \leq \frac{p-2}{3} ; \\
2, & \text { if } \frac{p-2}{3}+1 \leq j \leq p-1 ;
\end{array} \text { and } h\left(u_{p} u_{1}\right)=1 .\right. \\
& h\left(v_{j} v_{j+1}\right)=\left\{\begin{array}{ll}
0, & \text { if } 1 \leq j \leq \frac{q}{3} ; \\
2, & \text { if } \frac{q}{3}+1 \leq j \leq q-1 .
\end{array}, h\left(v_{q} u_{p}\right)=2 .\right.
\end{aligned}
$$

Case 3.3 If $p \neq 0(\bmod 3)$ and $q \neq 0(\bmod 3)$. We define $h: E_{G} \rightarrow\{0,1,2\}$ as:

$$
\begin{aligned}
& h\left(u_{j} u_{j+1}\right)=\left\{\begin{array}{ll}
0, & \text { if } 1 \leq j \leq \frac{p-1}{3} ; \\
2, & \text { if } \frac{p-1}{3}+1 \leq j \leq p-1 ;
\end{array} \text { and } h\left(u_{p} u_{1}\right)=1 .\right. \\
& h\left(v_{j} v_{j+1}\right)=\left\{\begin{array}{ll}
0, & \text { if } 1 \leq j \leq \frac{q-1}{3} \\
2, & \text { if } \frac{q-1}{3}+1 \leq j \leq q-1 .
\end{array}, h\left(v_{q} u_{p}\right)=2 .\right.
\end{aligned}
$$

So we obtain $\operatorname{sum}(0)=2 t+2, \operatorname{sum}(1)=\operatorname{sum}(2)=2 t+1$. Thus $\left|\operatorname{sum}\left(x_{1}\right)-\operatorname{sum}\left(x_{2}\right)\right| \leq 1$ for $0 \leq x_{1}<$ $x_{2} \leq 2$. Hence $h$ is 3-TEPC labeling as discussed in Figure 6 .

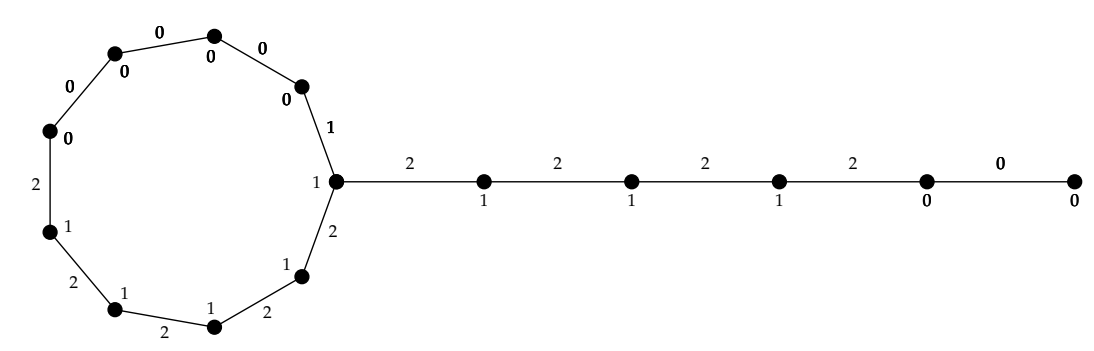

Figure 6. 3-TEPC labeling of $T_{9,5}$

Theorem 2. The book graph $B_{q}$ has 3-TEPC labeling.

Proof. Let $V_{G}=\left\{u, v, u_{j}, v_{j}, 1 \leq j \leq q\right\}$ and $E_{G}=\left\{u u_{j}, 1 \leq j \leq q\right\} \cup\left\{v v_{j}, 1 \leq j \leq q\right\} \cup\left\{u_{j} v_{j}, 1 \leq j \leq\right.$ $q\} \cup\{u v\}$ as shown in Figure 2. Now we see the following three cases: 
Case 1 Let $q \equiv 0(\bmod 3)$ which implies $q=3 t$, for $t \geq 1$. We define $h: E_{G} \rightarrow\{0,1,2\}$ as:

Case 1.1 Now we define edge labeling if $t$ is even:

$$
\begin{aligned}
& h\left(u u_{j}\right)=\left\{\begin{array}{ll}
0, & \text { if } 1 \leq j \leq t ; \\
2, & \text { if } t+1 \leq j \leq 3 t .
\end{array}, h\left(u_{j} v_{j}\right)= \begin{cases}0, & \text { if } 1 \leq j \leq t-1 ; \\
1, & \text { if } t \leq j \leq 3 t .\end{cases} \right. \\
& h\left(v v_{j}\right)=\left\{\begin{array}{ll}
0, & \text { if } 1 \leq j \leq t ; \\
2, & \text { if } t+1 \leq j \leq \frac{3 t}{2} ; \\
1, & \text { if } \frac{3 t}{2}+1 \leq j \leq 3 t .
\end{array}, h(u v)=2 .\right.
\end{aligned}
$$

Case 1.2 Now we define edge labeling if $t$ is odd:

$$
\begin{aligned}
& h\left(u u_{j}\right)=\left\{\begin{array}{ll}
0, & \text { if } 1 \leq j \leq t ; \\
2, & \text { if } t+1 \leq j \leq 3 t .
\end{array}, h\left(u_{j} v_{j}\right)= \begin{cases}0, & \text { if } 1 \leq j \leq t-1 ; \\
1, & \text { if } t \leq j \leq 3 t .\end{cases} \right. \\
& h\left(v v_{j}\right)=\left\{\begin{array}{ll}
0, & \text { if } 1 \leq j \leq t ; \\
2, & \text { if } t+1 \leq j \leq \frac{3 t+1}{2} ; \\
1, & \text { if } \frac{3 t+1}{2}+1 \leq j \leq 3 t .
\end{array}, h(u v)=1 .\right.
\end{aligned}
$$

So we obtain $\operatorname{sum}(0)=\operatorname{sum}(1)=\operatorname{sum}(2)=5 t+1$. Thus $\left|\operatorname{sum}\left(x_{1}\right)-\operatorname{sum}\left(x_{2}\right)\right| \leq 1$ for $0 \leq x_{1}<x_{2} \leq 2$. Hence $h$ is 3-TEPC labeling.

Case 2 Let $q \equiv 1(\bmod 3)$ which implies $q=3 t+1$, for $t \geq 1$. We define $h: E_{G} \rightarrow\{0,1,2\}$ as:

Case 2.1 Now we define edge labeling if $t$ is even:

$$
\begin{aligned}
& h\left(v v_{j}\right)=\left\{\begin{array}{ll}
0, & \text { if } 1 \leq j \leq t ; \\
2, & \text { if } t+1 \leq j \leq 3 t+1 .
\end{array}, h\left(u_{j} v_{j}\right)= \begin{cases}0, & \text { if } 1 \leq j \leq t ; \\
1, & \text { if } t+1 \leq j \leq 3 t+1 .\end{cases} \right. \\
& h\left(u u_{j}\right)=\left\{\begin{array}{ll}
0, & \text { if } 1 \leq j \leq t ; \\
2, & \text { if } t+1 \leq j \leq \frac{3 t}{2} ; \\
1, & \text { if } \frac{3 t}{2}+1 \leq j \leq 3 t+1 .
\end{array}, h(u v)=2 .\right.
\end{aligned}
$$

Case 2.2 Now we define edge labeling if $t$ is odd:

$$
\begin{aligned}
& h\left(v v_{j}\right)=\left\{\begin{array}{ll}
0, & \text { if } 1 \leq j \leq t ; \\
2, & \text { if } t+1 \leq j \leq 3 t+1 .
\end{array}, h\left(u_{j} v_{j}\right)= \begin{cases}0, & \text { if } 1 \leq j \leq t ; \\
1, & \text { if } t+1 \leq j \leq 3 t+1 .\end{cases} \right. \\
& h\left(u u_{j}\right)=\left\{\begin{array}{ll}
0, & \text { if } 1 \leq j \leq t ; \\
2, & \text { if } t+1 \leq j \leq \frac{3 t+1}{2} ; \\
1, & \text { if } \frac{3 t+1}{2}+1 \leq j \leq 3 t+1 .
\end{array}, h(u v)=1 .\right.
\end{aligned}
$$

So we obtain $\operatorname{sum}(0)=5 t+2, \operatorname{sum}(1)=\operatorname{sum}(2)=5 t+3$. Thus $\left|\operatorname{sum}\left(x_{1}\right)-\operatorname{sum}\left(x_{2}\right)\right| \leq 1$ for $0 \leq x_{1}<$ $x_{2} \leq 2$. Hence $h$ is 3 -TEPC labeling.

Case 3 Let $q \equiv 2(\bmod 3)$ which implies $q=3 t+2$, for $t \geq 1$. We define $h: E_{G} \rightarrow\{0,1,2\}$ as:

Case 3.1 Now we define edge labeling if $t$ is even:

$$
\begin{aligned}
& h\left(v v_{j}\right)=\left\{\begin{array}{ll}
0, & \text { if } 1 \leq j \leq t ; \\
2, & \text { if } t+1 \leq j \leq 3 t+2 .
\end{array}, h\left(u_{j} v_{j}\right)= \begin{cases}0, & \text { if } 1 \leq j \leq t ; \\
1, & \text { if } t+1 \leq j \leq 3 t+2 .\end{cases} \right. \\
& h\left(u u_{j}\right)=\left\{\begin{array}{ll}
0, & \text { if } 1 \leq j \leq t+1 ; \\
2, & \text { if } t+2 \leq j \leq \frac{3 t}{2}+1 ; \\
1, & \text { if } \frac{3 t}{2}+2 \leq j \leq 3 t+2 .
\end{array}, h(u v)=0 .\right.
\end{aligned}
$$


Case 3.2 Now we define edge labeling if $t$ is odd:

$$
\begin{aligned}
& h\left(v v_{j}\right)=\left\{\begin{array}{ll}
0, & \text { if } 1 \leq j \leq t ; \\
2, & \text { if } t+1 \leq j \leq 3 t+2 .
\end{array}, h\left(u_{j} v_{j}\right)= \begin{cases}0, & \text { if } 1 \leq j \leq t+1 ; \\
1, & \text { if } t+2 \leq j \leq 3 t+2 .\end{cases} \right. \\
& h\left(u u_{j}\right)=\left\{\begin{array}{ll}
0, & \text { if } 1 \leq j \leq t ; \\
2, & \text { if } t+1 \leq j \leq \frac{3 t+1}{2} ; \\
1, & \text { if } \frac{3 t+1}{2}+1 \leq j \leq 3 t+2 .
\end{array}, h(u v)=2 .\right.
\end{aligned}
$$

So we obtain $\operatorname{sum}(0)=5 t+5, \operatorname{sum}(1)=\operatorname{sum}(2)=5 t+4$. Thus $\left|\operatorname{sum}\left(x_{1}\right)-\operatorname{sum}\left(x_{2}\right)\right| \leq 1$ for $0 \leq x_{1}<$ $x_{2} \leq 2$. Hence $h$ is 3 -TEPC labeling.

Example 1. The graph $B_{6}$ and its given 3-TEPC labeling as shown in Figure 7.

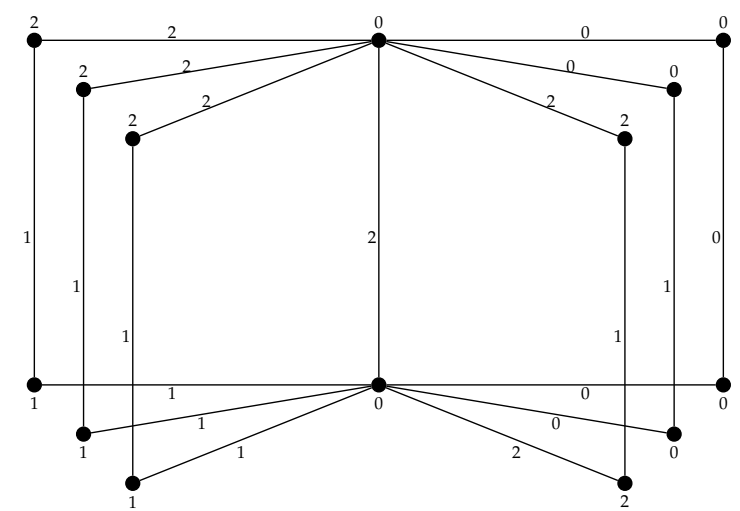

Figure 7. 3-TEPC labeling of $B_{6}$

Theorem 3. The flower graph $F l_{q}$ has 3-TEPC labeling.

Proof. Let $V_{G}=\left\{u, u_{j}, v_{j}, 1 \leq j \leq q\right\}$ and $E_{G}=\left\{u u_{j}, 1 \leq j \leq q\right\} \cup\left\{u_{j} u_{j+1}, 1 \leq j \leq q-1\right\} \cup\left\{u_{j} v_{j}, 1 \leq j \leq\right.$ $q\} \cup\left\{u_{j+1} v_{j}, 1 \leq j \leq q-1\right\} \cup\left\{u_{q} u_{1}\right\} \cup\left\{u_{1} v_{q}\right\}$ as shown in Figure 3 . Now we see the following three cases:

Case 1 Let $q \equiv 0(\bmod 3)$ which implies $q=3 t$, for $t \geq 1$. We define $h: E_{G} \rightarrow\{0,1,2\}$ as:

$$
\begin{aligned}
& h\left(u_{j} u_{j+1}\right)=\left\{\begin{array}{ll}
0, & \text { if } 1 \leq j \leq t ; \\
1, & \text { if } t+1 \leq j \leq 3 t-1 ;
\end{array} \text { and } h\left(u_{3 t} u_{1}\right)=1 .\right. \\
& h\left(u_{j+1} v_{j}\right)=\left\{\begin{array}{ll}
0, & \text { if } 1 \leq j \leq t-1 ; \\
2, & \text { if } t \leq j \leq 3 t-1 ;
\end{array} \text { and } h\left(u_{1} v_{3 t}\right)=2 .\right. \\
& h\left(u u_{j}\right)=\left\{\begin{array}{ll}
0, & \text { if } 1 \leq j \leq t+1 ; \\
2, & \text { if } t+2 \leq j \leq 2 t ; \\
1, & \text { if } 2 t+1 \leq j \leq 3 t .
\end{array}, h\left(u_{j} v_{j}\right)= \begin{cases}0, & \text { if } 1 \leq j \leq t-1 ; \\
2, & \text { if } t \leq j \leq 3 t .\end{cases} \right.
\end{aligned}
$$

So we obtain $\operatorname{sum}(1)=6 t+1, \operatorname{sum}(0)=\operatorname{sum}(2)=5 t$. Thus $\left|\operatorname{sum}\left(x_{1}\right)-\operatorname{sum}\left(x_{2}\right)\right| \leq 1$ for $0 \leq x_{1}<x_{2} \leq$ 2 . Hence $h$ is 3-TEPC labeling.

Case 2 Let $q \equiv 1(\bmod 3)$ which implies $q=3 t+1$, for $t \geq 1$. We define $h: E_{G} \rightarrow\{0,1,2\}$ as:

$$
h\left(u_{j} u_{j+1}\right)=\left\{\begin{array}{ll}
0, & \text { if } 1 \leq j \leq t ; \\
1, & \text { if } t+1 \leq j \leq 3 t
\end{array} \text { and } h\left(u_{j} u_{3 t+1}\right)=1 .\right.
$$




$$
\begin{aligned}
& h\left(u_{j+1} v_{j}\right)=\left\{\begin{array}{ll}
0, & \text { if } 1 \leq j \leq t ; \\
2, & \text { if } t+1 \leq j \leq 3 t ;
\end{array} \text { and } h\left(u_{1} v_{3 t+1}\right)=2 .\right. \\
& h\left(u u_{j}\right)=\left\{\begin{array}{ll}
0, & \text { if } 1 \leq j \leq t+1 ; \\
2, & \text { if } t+2 \leq j \leq 2 t+1 ; \\
1, & \text { if } 2 t+2 \leq j \leq 3 t+1 .
\end{array}, h\left(u_{j} v_{j}\right)= \begin{cases}0, & \text { if } 1 \leq j \leq t ; \\
2, & \text { if } t+1 \leq j \leq 3 t+1 .\end{cases} \right.
\end{aligned}
$$

So we obtain $\operatorname{sum}(0)=6 t+3, \operatorname{sum}(1)=\operatorname{sum}(2)=5 t+2$. Thus $\left|\operatorname{sum}\left(x_{1}\right)-\operatorname{sum}\left(x_{2}\right)\right| \leq 1$ for $0 \leq x_{1}<$ $x_{2} \leq 2$. Hence $h$ is 3 -TEPC labeling.

Case 3 Let $q \equiv 2(\bmod 3)$ which implies $q=3 t+2$, for $t \geq 1$. We define $h: E_{G} \rightarrow\{0,1,2\}$ as:

$$
\begin{aligned}
& h\left(u_{j} u_{j+1}\right)=\left\{\begin{array}{ll}
0, & \text { if } 1 \leq j \leq t ; \\
2, & \text { if } t+1 \leq j \leq 3 t+1 ;
\end{array} \text { and } h\left(u_{3 t+2} u_{1}\right)=2 .\right. \\
& h\left(u_{j+1} v_{j}\right)=\left\{\begin{array}{ll}
0, & \text { if } 1 \leq j \leq t ; \\
1, & \text { if } t+1 \leq j \leq 3 t+1 ;
\end{array} \text { and } h\left(u_{1} v_{3 t+2}\right)=1 .\right. \\
& h\left(u u_{j}\right)=\left\{\begin{array}{ll}
0, & \text { if } 1 \leq j \leq t+1 ; \\
2, & \text { if } t+2 \leq j \leq 3 t+2 .
\end{array}, h\left(u_{j} v_{j}\right)= \begin{cases}0, & \text { if } 1 \leq j \leq t+1 ; \\
1, & \text { if } t+2 \leq j \leq 3 t+2 .\end{cases} \right.
\end{aligned}
$$

So we obtain $\operatorname{sum}(0)=6 t+5, \operatorname{sum}(1)=\operatorname{sum}(2)=5 t+4$. Thus $\left|\operatorname{sum}\left(x_{1}\right)-\operatorname{sum}\left(x_{2}\right)\right| \leq 1$ for $0 \leq x_{1}<$ $x_{2} \leq 2$. Hence $h$ is 3 -TEPC labeling.

Example 2. The graph $\mathrm{Fl}_{6}$ and its given 3-TEPC labeling as shown in Figure 8.

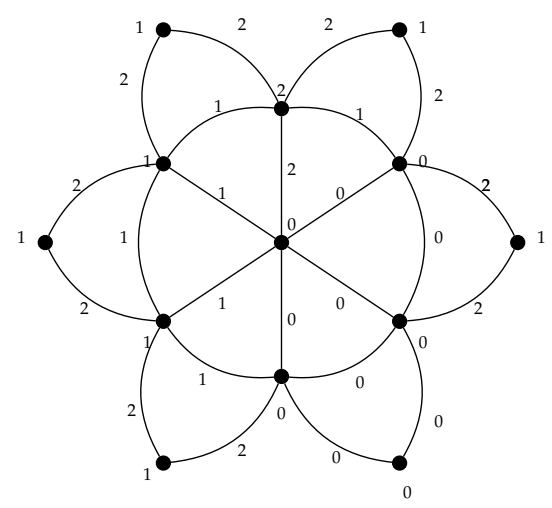

Figure 8. 3-TEPC labeling of $F l_{6}$

Acknowledgments: This article has benefited from constructive reviewing. Any remaining errors are my own

Author Contributions: All authors contributed equally to the writing of this paper. All authors read and approved the final manuscript.

Conflicts of Interest: "The authors declare no conflict of interest."

\section{References}

[1] West, D. B. (1996). Introduction to graph theory (Vol. 2). Upper Saddle River, NJ: Prentice hall.

[2] Cahit, I. (1987). Cordial graphs-a weaker version of graceful and harmonious graphs. Ars Combinatoria,23, 201-207.

[3] Sundaram, M., Ponraj, R., \& Somasundaram, S. (2004). Product cordial labeling of graphs. Bull. Pure and Applied Sciences (Mathematics \& Statistics), E, 23, 155-163.

[4] Ponraj, R., Sundaram, M., \& Sivakumar, M. (2012). K-total product cordial labelling of graphs. Applications and Applied Mathematics, 7, 708-716. 
[5] Sundaram, M., Ponraj, R., \& Somasundram, S. (2006). Total product cordial labeling of graphs. Bulletin of Pure $\mathcal{E}$ Applied Sciences. Sec. E, Mathematics E Statistics, 25, 199-203.

[6] Vaidya, S. K., \& Barasara, C. M. (2012). Edge product cordial labeling of graphs. Journal of Mathematical and computational Science, 2(5), 1436-1450.

[7] Vaidya, S. K., \& Barasara, C. M. (2013). Total edge product cordial labeling of graphs. Malaya Journal of Matematik, 3(1), 55-63.

[8] Azaizeh, A., Hasni, R., Ahmad, A., \& Lau, G. C. (2015). 3-Totat edge product cordial labeling of graphs. Far East journal of Matheimatical sciences, 96(2), 193-209.

[9] Ivanco, J. (2017). On 3-total edge product cordial connected graphs. Opuscula Mathematica, 37(5), 725-734.

[10] Yan, L., Li, Y., Zhang, X., Saqlain, M., Zafar, S., \& Farahani, M. R. (2018). 3-total edge product cordial labeling of some new classes of graphs. Journal of Information and Optimization Sciences, 39(3), 705-724.

[11] Ahmad, Y., Ali, U., Zafar, S., \& Zahid, Z. (2017). Some new standard graphs labeled by 3-total edge product cordial labeling. Applied Mathematics and Nonlinear Sciences, 2(1), 61-72.

[12] Ali, U., Bilal, M., Zafar, S., \& Zahid, Z. (2017). Some Families of Convex Polytopes Labeled by 3-Total Edge Product Cordial Labeling. Punjab University Journal of Mathematics, 49, 119-132.

[13] Ahmad, A., Baća, M., Naseem, M., \& Semanicová-Fenovcíková, A. (2017). On 3-total edge product cordial labeling of honeycomb. AKCE International Journal of Graphs and Combinatorics, 14(2), 149-157.

[14] Ali, U., Iqbal, J., Zafar, S., \& Zahid, Z. (2018). 4-total edge product cordial labeling of some standard graphs. Tamap Journal of Mathematics and Statistics, 16-31.

[15] Ivanco, J. (2017). On k-total edge product cordial graphs. The Australasian Journal of Combinatorics, 67, 476-485.

[16] Hasni, R., \& Azaizeh, A. (2016). 3-total edge product cordial labeling of wheel related graphs. Matematika, 32(2),

[17] Kuo, D., Chang, G. J., \& Kwong, Y. H. (1997). Cordial labeling of $m K_{n}$. Discrete Mathematics, 169(1-3), $121-131$.

[18] Lai, Y. L., \& Chen, Y. M., On the total product cordial labeling of $P_{n}+m K_{1}$. National Chiayi University. http:/ / people.chu.edu.tw/ algo2008/file/f55.pdf

(C) 2020 by the author; licensee PSRP, Lahore, Pakistan. This article is an open access article distributed under the terms and conditions of the Creative Commons Attribution (CC-BY) license (http://creativecommons.org/licenses/by/4.0/). 